\title{
Actualización sobre la biología molecular de los gliomas: hacia una clasificación patomolecular de los gliomas
}

\author{
M.A. Idoate, J. Echeveste
}

\author{
ACTUALIZACIÓN SOBRE LA BIOLOGÍA MOLECULAR DE LOS GLIOMAS: \\ HACIA UNA CLASIFICACIÓN PATOMOLECULAR DE LOS GLIOMAS
}

\begin{abstract}
Resumen. Objetivo. Revisar el estado actual de la clasificación y oncogénesis de los gliomas, enfatizando aquellos factores biológicos con especial significación clínica. Desarrollo. En la clasificación actual, la gradación histológica de los gliomas, junto con el diagnóstico del tipo histológico, son los parámetros anatomopatológicos de mayor impacto clínico. Ambos factores constituyen una obligada referencia para todo estudio molecular y son la base para una nueva clasificación. La asociación de la estirpe oligodendroglial con la pérdida de las regiones cromosómicas 1p y 19q es muy útil en el diagnóstico diferencial histopatológico. La nueva clasificación patomolecular de los gliomas deberá considerar las sendas biológicas de malignización de los gliomas, ya que tienen un carácter pronóstico. Esta clasificación también deberá establecer la potencialidad biológica del tumor en función de la heterogeneidad, buscando estrategias originales como partir de material obtenido mediante microdisección. Si bien en la actualidad no existen perfiles moleculares pronósticos, ciertas alteraciones como la deleción del cromosoma 10, y en especial de la región 10q23, la mutación de los genes PTEN y TP53 y la amplificación o mutación del gen EGFR, resultan especialmente relevantes. De cara al tratamiento, la deleción combinada $1 p / 19 q$ permite identificar a aquellos oligodendrogliomas anaplásicos especialmente quimiosensibles. Conclusiones. La nueva clasificación patomolecular de los gliomas, que no sustituirá sino que complementará a la actual, deberá considerar la oncogénesis y heterogeneidad de los gliomas. Esta nueva clasificación deberá tener aplicación clínica e integrar los nuevos hallazgos moleculares con los datos histopronósticos básicos. [REV NEUROL 2007; 44: 217-24]
\end{abstract}

Palabras clave. Alteración molecular. Astrocitomas. Factores pronósticos. Gliomas. Inmunohistoquímica. Oncogénesis.

\section{INTRODUCCIÓN}

Los gliomas son tumores de origen glial o neuroepitelial. Con este término se agrupan los astrocitomas, los oligodendrogliomas y los ependimomas. Los gliomas son los tumores primarios más frecuentes del sistema nervioso, que afectan a niños y adultos. La incidencia poblacional estimada en nuestro medio de los gliomas malignos es de aproximadamente 4 casos por 100.000 habitantes/año. Si bien la incidencia poblacional de los gliomas no es muy alta en términos relativos, la falta de un tratamiento eficaz supone un problema médico de gran importancia. Además, los gliomas, que representan el $71 \%$ de los tumores intracraneales de los pacientes menores de 9 años, suponen el grupo de tumores sólidos con más prevalencia en este grupo de edad.

El objetivo de este trabajo es una actualización de los avances en la investigación molecular en gliomas, incluyendo una visión personal del problema por los autores. En la actualidad se conoce mucho mejor la oncogénesis de los gliomas. Además, nuevos datos pronósticos de índole molecular han pasado ya a la práctica clínica. Por tanto, no se pretende tratar todos los factores moleculares sobre los que se investiga en la actualidad, sino comentar fundamentalmente aquellas alteraciones moleculares a las que los datos actuales otorgan una mayor significación clínica.

Aceptado tras revisión externa: 12.01.07.

Departamento de Anatomía Patológica. Clínica Universitaria. Facultad de Medicina. Universidad de Navarra. Pamplona, Navarra, España.

Correspondencia: Dr. Miguel Ángel Idoate Gastearena. Departamento de Anatomía Patológica. Clínica Universitaria. Facultad de Medicina. Universidad de Navarra. Avda. Pío XII, s/n. E-31008 Pamplona (Navarra). E-mail: maidoate@unav.es

(C) 2007, REVISTA DE NEUROLOGÍA

\section{CLASIFICACIÓN ACTUAL DE LOS ASTROCITOMAS Tipo histopatológico}

La clasificación de los tumores cerebrales ha estado y está basada en criterios histopatológicos. Desde la clasificación de Bailey y Cushing de los tumores cerebrales (1928) hasta la actual de la Organización Mundial de la Salud (OMS) [1] han pasado más de 70 años de sucesivas aportaciones debidas a un mejor conocimiento de los rasgos histológicos e inmunohistoquímicos de los tumores. Esto ha permitido establecer diferentes grupos tumorales histogenéticos y comprender mejor la oncopatología del sistema nervioso. Se ha demostrado que existe una clara relación entre el tipo histopatológico y el pronóstico. Así, se sabe que los astrocitomas muestran un comportamiento más agresivo que los oligodendrogliomas o ependimomas.

\section{Grado histopatológico}

Un paso posterior fue la gradación de los tumores de acuerdo a la agresividad biológica. Se establecieron así los grados histológicos pronósticos de la OMS (grados I a IV). Esta clasificación ha sido especialmente útil en el caso de los gliomas (Tabla I). La gradación de los gliomas se basa en unos parámetros histológicos clave, que se discuten en los siguientes apartados.

\section{ASPECTOS GENERALES DE LOS ASTROCITOMAS}

La supervivencia media de los pacientes afectos de astrocitoma es muy diferente en función del grado tumoral: 10-12 meses para el glioblastoma multiforme, 24 meses en el caso del astrocitoma anaplásico, y 6-8 años en los astrocitomas de bajo grado. Por otra parte, la supervivencia de los pacientes afectos de glioblastoma es diferente según se trate de glioblastomas secundarios o primarios [2]. La supervivencia en los astrocitomas de bajo grado es muy variable, dependiendo de una diversidad de fac- 
tores como la localización del tumor, la edad (mayor o menor de 40 años), la valoración del estado funcional del paciente mediante el índice de Karnofsky, el grado de resección del tumor y, de manera muy especial, el grado de malignización potencial del astrocitoma, que depende en última instancia del grado de estabilidad o inestabilidad genómica. Esto explica que haya astrocitomas que malignizan uno o dos años después de la intervención quirúrgica frente a otros que no malignizan en un período de más de 10 años.

La correcta gradación de los astrocitomas tiene un significado pronóstico y terapéutico. La gradación actual de los astrocitomas se estableció en función de unos parámetros histológicos clave, como la densidad celular, la atipia nuclear, la presencia de figuras mitóticas, el desarrollo de vasos anómalos y la necrosis. Existen varias clasificaciones, siendo la más utilizada la de la OMS, que los divide en grados I a IV. El seguimiento de los pacientes y el estudio histopatológico de las biopsias de los astrocitomas permitió conocer el proceso de malignización, que procede de grados bajos a al-

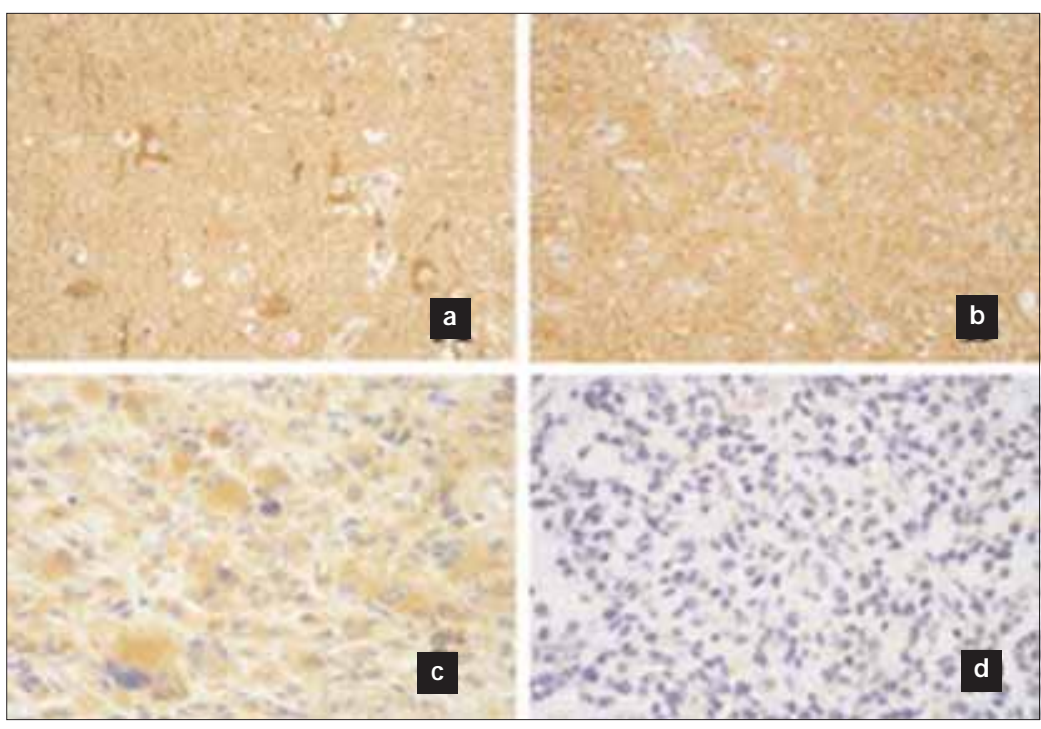

Figura 1. Expresión de la proteína DCC en tejido normal y astrocitoma. Se aprecia que la expresión de esta proteína es muy marcada en la corteza, especialmente en las neuronas (a). En los astrocitomas de grado II, la expresión es también intensa (b). En los glioblastomas primarios, la expresión es también marcada (c), mientras que en los glioblastomas secundarios tiende a perderse (d). Inmunocitoquímica frente a proteína DCC, $\times 200$. tos, de manera rápida o lenta, sin aparente tendencia hacia la maduración (regresión en el grado histológico).

La valoración de algunos de estos parámetros histológicos se presta a la subjetividad y al azar. Esto ocurre especialmente con la atipia, la proliferación vascular y la identificación de figuras mitóticas -que pueden ser muy escasas-. También hay que considerar los errores de gradación que se producen en función del muestreo, de la cantidad de tumor extirpado -resección del tumor frente a biopsia estereotáxica- y de la propia heterogeneidad intratumoral, que puede ser marcada $[3,4]$. Por eso, la gradación del tumor debe hacerse siempre en las áreas de mayor grado de agresividad histológica.

Estos rasgos morfológicos reflejan aspectos biológicos clave como la proliferación celular, la alteración del ADN y la angiogénesis. Las moléculas que intervienen en estas alteraciones biológicas son abordables mediante técnicas de biología molecular. Así se ha conocido qué alteraciones moleculares se producían y cuál era su relación con el proceso de malignización morfológica. Por ello, la gradación morfológica de la malignización de los astrocitomas ha sido clave para entender las alteraciones moleculares.

\section{CLASIFICACIÓN MOLECULAR DE LOS ASTROCITOMAS}

Un problema importante de la nueva clasificación es el desconocimiento de la etiopatogenia de los astrocitomas. Se estima que un $5 \%$ de los astrocitomas cerebrales son hereditarios, asociados especialmente a la neurofibromatosis [5]. Un factor etiológico adquirido demostrado en los astrocitomas es la radiación.

Del estudio del genoma de los astrocitomas se puede concluir que existe todavía un gran número de alteraciones genéticas de significado desconocido, como se deduce de los estudios de hibridación genómica comparada. Las alteraciones citogenéticas detectadas mediante esta técnica son del tipo de amplificación y deleción, y en el caso de los astrocitomas se ha visto que afectan a prácticamente la totalidad de los cromosomas. A esto hay que añadir las alteraciones debidas a mutaciones puntuales y a cambios epigenéticos como la hipermetilación de genes promotores.

Se ha observado que existen unas alteraciones moleculares propias de cada grado histológico tumoral. De forma paralela al proceso de malignización histológica, existe una secuencia de eventos genéticos, que incluyen la amplificación del gen $P D G F / R$ y la mutación del gen TP53 como acontecimientos moleculares iniciales, y la alteración de los genes EGFR y PTEN como alteraciones tardías.

La investigación molecular actual ha demostrado que en los astrocitomas hereditarios existen unos genes alterados causantes del desarrollo tumoral, que se conocen con bastante aproximación (Tabla II). Se trata de unas proteínas activadoras de la GTPasa en la neurofibromatosis (17q11 y 22q12), del complejo responsable de las reparaciones de ADN en el síndrome de Turcot (3p21 y 7p22) y de la proteína p53 en el síndrome de Li-Fraumeni (17p13).

\section{El gran problema de la heterogeneidad molecular}

Un aspecto fundamental a tener en cuenta de los astrocitomas es la heterogeneidad fenotípica y molecular, más marcada cuánto más maligno es el tumor. La neoplasia puede presentar distintas propiedades biológicas en diferentes áreas del tumor en función de los clones tumorales que lo componen. Este hecho dificulta más la de por sí ardua tarea de clasificación biológica tumoral. En un estudio propio de una serie de astrocitomas de grado II se apreció que la actividad proliferativa tumoral era baja en la mayoría del tumor, pero que existían algunos casos en los que coexistía con pequeños focos de mayor actividad proliferativa, mayor densidad celular y pérdida de la expresión de proteínas importantes en la oncogénesis, como es la proteína DCC (Fig. 1). Por el seguimiento de los pacientes y la realización de nuevas biopsias se comprobó que estos focos eran indicativos de un cambio en el grado tumoral.

La heterogeneidad intratumoral en los astrocitomas es un hecho relevante. Esto se ha descrito en relación con los rasgos histológicos, la actividad proliferativa y alteraciones génicas como, 
Tabla I. Gradación histológica, denominación y características generales de los gliomas: clasificación de la Organización Mundial de la Salud (OMS) y clasificación St. Anne-Mayo.

\begin{tabular}{llll}
\hline Clasificación OMS & Clasificación St. Anne-Mayo & Otra denominación & Características generales \\
\hline Grado I & & $\begin{array}{l}\text { Ganglioglioma. } \\
\text { Astrocitoma pilocítico }\end{array}$ & $\begin{array}{l}\text { Benigno. Bien delimitado. } \\
\text { Niños y jóvenes }\end{array}$ \\
\hline $\begin{array}{l}\text { Grado II. Ligera hipercelularidad, } \\
\text { no o moderada atipia nuclear, } \\
\text { no (u ocasional) figuras mitóticas }\end{array}$ & $\begin{array}{l}\text { Grado 1 o 2. Un criterio } \\
\text { (generalmente atipia nuclear) }\end{array}$ & $\begin{array}{l}\text { Astrocitoma (de bajo grado) } \\
\text { Oligodendroglioma (de bajo grado) } \\
\text { Oligoastrocitoma de bajo grado } \\
\text { Ependimoma (de bajo grado) }\end{array}$ & $\begin{array}{l}\text { Relativamente benigno. Infiltrante. } \\
\text { Recidivante. Niños y jóvenes }\end{array}$ \\
\hline $\begin{array}{l}\text { Grado III. Marcada hipercelularidad, } \\
\text { atipia nuclear, mitosis frecuentes }\end{array}$ & $\begin{array}{l}\text { Grado 3. Un criterio } \\
\text { (generalmente atipia nuclear) }\end{array}$ & $\begin{array}{l}\text { Astrocitoma anaplásico o astrocitoma } \\
\text { de alto grado. Oligodendroglioma } \\
\text { anaplásico. Oligoastrocitoma } \\
\text { anaplásico. Ependimoma anaplásico }\end{array}$ & $\begin{array}{l}\text { Maligno. Infiltrante. Rápidamente } \\
\text { recidivante. Adultos }\end{array}$ \\
\hline $\begin{array}{l}\text { Grado IV. Marcada hipercelularidad, } \\
\text { atipia nuclear, mitosis, necrosis }\end{array}$ & $\begin{array}{l}\text { Grado 4. Un criterio } \\
\text { (generalmente atipia nuclear) }\end{array}$ & $\begin{array}{l}\text { Glioblastoma multiforme o } \\
\text { astrocitoma de alto grado: de novo } \\
\text { o secundario. Gliosarcoma }\end{array}$ & $\begin{array}{l}\text { Maligno. Infiltrante. Rápidamente } \\
\text { recidivante. Potencialmente } \\
\text { metastatizante. Adultos }\end{array}$ \\
\hline \begin{tabular}{l} 
Criterios anatomopatológicos clave: densidad celular, atipia nuclear, presencia de figuras mitóticas, necrosis tumoral, proliferación vascular atípica. \\
\hline
\end{tabular} & & &
\end{tabular}

Tabla II. Síndromes clínicos causantes de tumores hereditarios del sistema nervioso y sus alteraciones moleculares correspondientes.

\begin{tabular}{lll}
\hline Síndrome clínico / Tumores del sistema nervioso que originan & $\begin{array}{l}\text { Alteraciones génicas } \\
\text { responsables / Localización }\end{array}$ & Función de las proteínas alteradas \\
\hline $\begin{array}{l}\text { Síndrome de la poliposis adenomatosa familiar colorrectal } \\
\text { (síndrome de Turcot) / M eduloblastoma, gliomas }\end{array}$ & $\begin{array}{l}\text { HMLH1 / 3p21 } \\
H P S M 2 \text { / 7p22 }\end{array}$ & $\begin{array}{l}\text { Proteínas del complejo responsable } \\
\text { de las reparaciones de ADN }\end{array}$ \\
\hline $\begin{array}{l}\text { Síndrome de Li-Fraumeni / Gliomas, meduloblastoma, } \\
\text { tumores de plexos coroideos }\end{array}$ & TP53 / 17p13 & $\begin{array}{l}\text { Proteína que induce la parada del ciclo } \\
\text { celular y apoptosis }\end{array}$ \\
\hline $\begin{array}{l}\text { Neurofibromatosis tipos I y II / Gliomas, meningiomas, } \\
\text { tumores del sistema nervioso periférico, paragangliomas }\end{array}$ & $\begin{array}{l}\text { NF-1 / 17q11 } \\
\text { NF-2 / 22q12 }\end{array}$ & $\begin{array}{l}\text { Proteína (neurofibromina) activadora de la GTPasa } \\
\text { dependiente de ras }\end{array}$ \\
\hline $\begin{array}{l}\text { Proteína (merlina) que interacciona con la molécula } \\
\text { CD44 y la actina }\end{array}$ \\
$\begin{array}{l}\text { Esclerosis tuberosa / Astrocitoma de células gigantes } \\
\text { subependimario, hamartomas }\end{array}$ & $\begin{array}{l}\text { TSC1 / 9q34 } \\
\text { TSC2 / 16p13 }\end{array}$ & $\begin{array}{l}\text { Proteína (hamartina) de función desconocida } \\
\text { Proteína (tuberina) con homología con el dominio } \\
\text { catalítico de la GTP-asa }\end{array}$ \\
\hline
\end{tabular}

por ejemplo, el número de deleciones y amplificaciones de regiones cromosómicas, que eran distintas según el área tumoral estudiada [4,6]. Una estrategia interesante para el estudio de la heterogeneidad tumoral se basa en la microdisección tisular manual o mediante láser, seleccionando aquellas áreas que muestran especiales rasgos histológicos o inmunocitoquímicos, y la posterior obtención de $\mathrm{ADN}, \mathrm{ARN}$ o proteínas [7,8]. Es previsible que en el futuro se avance más en el conocimiento de las alteraciones moleculares referidas a áreas tumorales concretas, que permitan establecer si el tumor ha adquirido propiedades nuevas que lo doten de una mayor agresividad biológica y clínica.

\section{Astrocitomas genéticamente estables e inestables}

Hasta el momento no es posible predecir qué astrocitomas de bajo grado se transformarán, aunque existen datos moleculares útiles de cara a esta predicción. En este sentido podríamos distinguir, desde un punto de vista teórico, entre astrocitomas de bajo grado genéticamente estables (no se transforman) y genéticamente inestables (potencialidad de transformación).

Se propone la denominación de astrocitomas genéticamente estables para aquellos astrocitomas de grado II, y eventualmente de grado III, que no muestran tendencia a la adquisición de un grado histológico mayor. Por el contrario, podemos considerar como astrocitomas inestables a aquellos gliomas de grados II o III que muestran potencialidad para adquirir mayores gra- dos histológicos. Creemos que esta tendencia a la transformación puede valorarse mediante la evaluación inmunohistoquímica de determinados parámetros y de ciertas alteraciones génicas.

Los astrocitomas inestables no presentan por lo general rasgos histológicos distintivos, aunque pueden mostrar un componente gemistocítico notable. Se concibe al gemistocito como un tipo celular genéticamente inestable, con clara proclividad a malignizar. Los gemistocitos son astrocitos de citoplasma abundante, intensamente eosinofílico y núcleo excéntrico. Estas células son escasamente proliferantes, muestran una intensa expresión de la proteína fibrilar acídica glial (GFAP), tienen bloqueada la apoptosis y presentan mutación del gen TP53 [9]. Estos dos últimos hechos explicarían la especial susceptibilidad de los astrocitomas gemistocíticos -astrocitomas compuestos de al menos un $20 \%$ de gemistocitos- a malignizar. El inmunomarcaje de estas células mediante anticuerpos frente a Bcl-2 o GFAP es útil de cara a la estimación de la densidad de astrocitos gemistocíticos en el tumor.

Los aspectos más relevantes en la valoración de los astrocitomas de bajo grado inestables son:

- Marcadores de proliferación celular. Dos técnicas pueden aplicarse a la práctica diaria: inmunohistoquímica, especialmente frente al antígeno Ki-67, y citofluorometría. Parece preferible la evaluación inmunohistoquímica dado que la proliferación no tiene por qué ser homogénea en todo el teji- 
do tumoral. Un índice de proliferación mayor del 5\% frente a Ki-67 es indicativo de una proliferación inusual y se asocia a una mayor tendencia a la malignización. Por ello, la actividad proliferativa tumoral se correlaciona con la supervivencia en los astrocitomas de bajo grado [10,11]. Además, este marcador puede ser de ayuda en la asignación de grado en aquellos astrocitomas en los que se duda entre los grados II y III [12].

- Valor de la ploidía. La determinación de la ploidía en los astrocitomas de bajo grado tiene valor pronóstico [13], pero no se ha demostrado en los de alto grado. La aneuplodía en los astrocitomas de bajo grado va ligada a inestabilidad genómica, a la mutación del gen TP53 y a una mayor tendencia a la malignización [14].

- Detección de la inactivación de genes supresores y de la activación de oncogenes en las células tumorales. Existe una multiplicidad de factores moleculares sobre los que se realiza una intensa investigación [1]. De las alteraciones moleculares que se ha demostrado que intervienen en la malignización de los astrocitomas, ninguna parece tener valor pronóstico independiente de forma aislada [15-17]. Se ha descrito una multiplicidad de alteraciones moleculares, de las que cabe destacar la mutación del gen TP53, la deleción del cromosoma $17 \mathrm{p}$, la amplificación del cromosoma 7, la deleción del cromosoma 10, la mutación del gen PTEN (phosphatase and tensin homologue deleted from chromosome $T E N$ ), la deleción del gen DCC (gen delecionado en el cáncer de colon), la sobreexpresión, mutación o amplificación del gen EGFR (receptor del factor de crecimiento epidérmico) y la sobreexpresión del gen $V E G F$ (factor de crecimiento del endotelio vascular) y de su receptor. Otros genes implicados en el control del ciclo celular también están alterados, como el retinoblastoma, el P16, el MDM2, etc.

- Posible valor de la mutación del gen TP53 como indicador de astrocitomas de bajo grado inestables [18]. En los astrocitomas en los que el gen TP53 está mutado no se ejerce el doble efecto de bloqueo celular -a través del gen $P 21-$ y de facilitación de la apoptosis -a través del gen proapoptótico $B A X-$. La mutación del gen favorece por tanto que se produzcan clones de células tumorales proliferantes y refractarias a la apoptosis, sobre los cuales se desarrollan eventos génicos acumulativos responsables de la malignización. La detección inmunohistoquímica de la proteína p53 en los astrocitomas de bajo grado con valores relativos superiores al $20 \%$ de núcleos tumorales marcados se correlaciona con el daño del gen.

No hay que olvidar que existen mecanismos no asociados a la mutación del gen TP53 que estabilizan la proteína y ocasionan su acumulación [19]. Así, por ejemplo, existen tipos histológicos, como el astrocitoma pilocítico, que con frecuencia muestran una marcada inmunorreactividad para la proteína p53 no asociada a mutación del correspondiente gen [20]. Estas y otras razones explican por qué los estudios de correlación pronóstica sobre la base de la detección inmunohistoquímica de la proteína p53 en los astrocitomas de bajo grado han dado resultados contradictorios [21,22].

En resumen, se puede considerar que los astrocitomas de bajo grado aneuploides, inusualmente proliferativos y con el gen TP53 mutado, constituyen un subgrupo de tumores con una mayor tendencia a malignizar. Estas alteraciones por lo general tienden a ir unidas [14]. Además, los astrocitomas inestables presentan un mayor número de alteraciones genéticas. Desde el punto de vista citogenético y molecular, estos tumores presentan un mayor número de amplificaciones/deleciones que un astrocitoma estable [23].

\section{Astrocitomas grado IV (glioblastomas) primarios y secundarios. Una diferenciación necesaria}

Se consideran glioblastomas secundarios a aquellos astrocitomas de grado IV que proceden de grados histológicos menores, a diferencia de los primarios, que surgen directamente como tales. Los glioblastomas secundarios aparecen por lo general a una edad menor que los primarios ( $<45$ años). Es un hecho relevante que los glioblastomas secundarios tienen un mejor pronóstico que los primarios, y que son justamente los secundarios los diagnosticados en pacientes supervivientes a largo plazo [1]. La cuestión de si la mayor supervivencia de los glioblastomas secundarios se debe a la menor edad de los pacientes respecto a los primarios o a factores intrínsecos tumorales no se ha resuelto todavía.

Es difícil estimar qué porcentaje de los glioblastomas pertenece a uno u otro subtipo. Si bien los criterios teóricos son claros, en la identificación y clasificación de cada caso pueden generarse dudas, dada la escasez de muestra de que se dispone y la falta de datos clínicos. Según Ohgaki et al [24], en una serie de 715 glioblastomas, los glioblastomas secundarios no superaron el $5 \%$.

La diferenciación clínica o histológica entre ambos subtipos de glioblastoma es compleja. Como se ha indicado, se consideran glioblastomas secundarios a aquellos astrocitomas de grado IV que proceden de un grado menor, a diferencia de los primarios, que surgen directamente como tales. Por tanto, la demostración mediante sucesivas biopsias de la transformación del astrocitoma es un argumento definitivo de que se trata de un glioblastoma secundario. También la identificación de varios grados intratumorales (heterogeneidad de grado) constituye un argumento importante a favor de un glioblastoma secundario. Dado que el proceso de malignización puede ser subclínico, es posible que en el momento del diagnóstico todo el tumor sea de grado IV y haya anulado los grados previos, lo que dificultaría mucho su identificación como glioblastoma secundario.

Los glioblastomas primarios parecen tener un perfil molecular o citogenético distinto del de los secundarios, lo que indica que ambos subtipos de astrocitoma siguen vías oncogénicas distintas (Tabla III). Así, en el glioblastoma primario son frecuentes la amplificación de los genes EGFR y $M D M 2$, la mutación del gen $P T E N$ y la deleción homocigota del gen $P 16 / C D K N 2 A$. Por el contrario, la mutación del gen TP53/LOH $17 p$ y la deleción del gen $D C C$ son propias del glioblastoma secundario. Por lo tanto, la aplicación de este perfil molecular tiene utilidad en la subtipificación de los glioblastomas. En este diagnóstico diferencial es de interés la evaluación inmunohistoquímica de la proteína DCC. En un estudio propio de una serie de 37 glioblastomas, 27 primarios y 10 secundarios, observamos que el $70 \%$ de los secundarios eran inmunonegativos para la proteína DCC frente a sólo un $19 \%$ en los primarios (Fig. 2), hecho que se correlaciona con la pérdida del gen $D C C$ [25].

Los glioblastomas pediátricos y los inducidos por radiación presentan alteraciones moleculares similares a las propias de los glioblastomas secundarios, aunque se manifiestan clínica e histológicamente como glioblastomas primarios.

En el abordaje clínico de los astrocitomas se recomienda la realización de unas pruebas inmunohistoquímicas y molecula- 


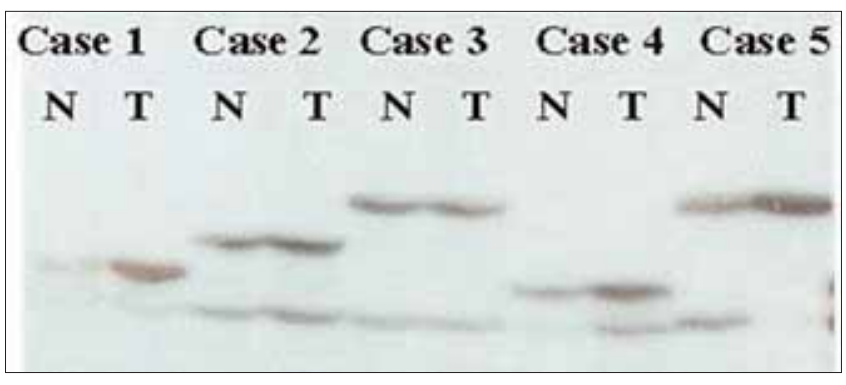

Figura 2. Deleción de la región cromosómica 18q21-23 (región DCC) LOH mediante una sonda específica que prueba un locus de microsatélites específicos (D18S69). Se aprecia deleción en los casos 1 y 5, que corresponden a un astrocitoma de grado III y a un astrocitoma de grado IV, respectivamente. N: tejido normal; T: tumor.

Tabla III. Características moleculares de los glioblastomas primarios frente a los secundarios.

\begin{tabular}{lll}
\hline Alteración génica/citogenética & $\begin{array}{l}\text { Glioblastoma } \\
\text { secundario }\end{array}$ & $\begin{array}{l}\text { Glioblastoma } \\
\text { primario }\end{array}$ \\
\hline Amplificación del gen EGFR & Raro & Frecuente \\
\hline Mutación del gen PTEN & Raro & Frecuente \\
\hline Amplificación del gen MDM2 & Raro & Frecuente \\
\hline $\begin{array}{l}\text { Deleción homocigota } \\
\text { del gen } P 16 / C D K N 2 A\end{array}$ & Raro & Frecuente \\
\hline Mutación del gen TP53/LOH 17p & Frecuente & Raro \\
\hline Deleción del gen $D C C$ & Frecuente & Raro \\
\hline
\end{tabular}

res que permiten estimar la potencialidad biológica de la neoplasia (Fig. 3).

\section{Importancia de la deleción de la región cromosómica $10 q$ en la nueva clasificación patomolecular}

El cromosoma 10 es clave en la oncogénesis de los astrocitomas. Frecuentemente puede perderse la región 10q, la región 10p o el cromosoma entero. La pérdida de heterocigosidad de la región $10 \mathrm{q}$ es la alteración molecular más frecuente en los glioblastomas. Los loci que con mayor frecuencia se afectan son el 10q23-24 y el 10q25-pter. En varios estudios se ha demostrado que la deleción de la región 10q23 es la única alteración genética con valor pronóstico independiente indicativo de una menor supervivencia [24,26,27]. En el locus 10q23-24 se localiza el gen PTEN y otros genes relevantes con una acción importante en el control de la proliferación celular, la invasión y la angiogénesis.

En un estudio propio [28] se encontró que la pérdida de heterocigosidad $(\mathrm{LOH})$ de la región cromosómica 10q23 (región PTEN) de los astrocitomas se asociaba a pérdida de la proteína y a un incremento en la actividad proliferativa tumoral. $\mathrm{La} \mathrm{LOH}$ de la región 10q23 era propia de los astrocitomas de alto grado, especialmente el glioblastoma. Sin embargo, ocasionalmente podía detectarse en astrocitomas de bajo grado, en especial en los tumores gemistocíticos.

La proteína PTEN se expresa en la corteza y la sustancia blanca normales, así como en el grado II, pero se pierde en los grados III y IV. Sin embargo, existe un subgrupo de astrocitomas de alto grado que, lejos de perder la proteína, parecen expresarla aún más intensamente que en el tejido cerebral normal de referencia.
Nuestro grupo ha llevado a cabo estudios de correlación entre la expresión inmunohistoquímica de la proteína PTEN y la valoración de la expresión del ARN mensajero (ARNm) del gen correspondiente mediante la técnica de reacción en cadena de la polimerasa y utilización de la transcriptasa inversa (RT-PCR) a tiempo real [29], utilizando como controles líneas celulares de glioblastoma con el PTEN mutado y tejido cerebral humano gliótico. En este estudio se observó que la expresión de ARNm del gen PTEN se correlacionaba con el grado tumoral. Por otra parte, sorprendentemente, los valores de RT-PCR del gen PTEN en los tumores inmunonegativos fueron similares a los de los tumores inmunopositivos. Por tanto, si la expresión de ARNm era similar en tumores inmunonegativos e inmunopositivos, la proteína PTEN detectada inmunocitoquímicamente podría ser una proteína acumulada, quizá estructuralmente anómala. Para resolver esta cuestión se requieren más estudios dirigidos a caracterizar esta proteína PTEN citoplasmática sobreabundante en las células tumorales.

\section{OLIGODENDROGLIOMAS Características generales}

Los oligodendrogliomas representan un 5-18\% de los gliomas. Pueden dividirse en oligodendrogliomas de bajo grado y anaplásicos. La incidencia poblacional estimada es de 0,3 casos por 100.000 habitantes/año. La supervivencia de los pacientes es distinta para los pacientes afectos de uno u otro de los subtipos tumorales citados. Algunos autores han apuntado que los pacientes afectos de oligodendrogliomas frontales tienen un mejor pronóstico.

\section{Dinámica de la malignización de los oligodendrogliomas}

La tendencia a la malignización de los oligodendrogliomas de bajo grado es frecuente, pero no tan acusada como en los astrocitomas. No es posible prever con seguridad con los medios actuales qué oligodendrogliomas malignizarán.

\section{Aspectos moleculares de interés en los oligodendrogliomas}

De manera resumida, los aspectos más relevantes son:

- Utilidad de la gradación histopatológica. Podemos diferenciar entre oligodendrogliomas de bajo grado (grado II) y anaplásicos (grado III). La tasa de supervivencia de los oligodendrogliomas de bajo grado a los cinco años, publicada por Shaw et al [30], fue del 58\%, y del 36\% para los anaplásicos. En otro estudio más reciente, la supervivencia para los oligodendrogliomas de bajo grado fue cercana al $78 \%$ a los cinco años, y casi del 30\% para los anaplásicos [31].

- La presencia de gemistocitos no marca una mayor proclividad a la malignización, a diferencia de lo que ocurre en los astrocitomas. En este sentido, Kros et al [32] mostraron que en su serie de pacientes con oligodendrogliomas, la diferenciación gemistocítica (microgemistocítica) no se asociaba a una menor supervivencia

- Utilidad de los marcadores de proliferación celular. Según algunos autores, valores de inmunorreactividad nuclear frente a Ki-67 mayores del 3\% en los oligodendrogliomas de bajo grado se correlacionan con un peor pronóstico [33].

- Valor de la ploidía. No se ha demostrado que la determinación de la ploidía tenga valor pronóstico [34].

- Detección de la mutación del gen TP53. Este hecho, junto con la detección inmunohistoquímica de la correspondiente 
proteína en los oligodendrogliomas de bajo grado, se asocia a un peor pronóstico, como sucede con los astrocitomas de bajo grado [35,36].

La deleción 1p o combinada $1 \mathrm{p} / 19 \mathrm{q}$ es una alteración citogenética propia de los oligodendrogliomas. De hecho, esta alteración resulta de utilidad en el diagnóstico diferencial entre astrocitomas y oligodendrogliomas [37] y es más frecuente en los oligodendrogliomas cerebrales que no se asientan en el lóbulo temporal. Quizá este hecho tenga relación con el postulado mejor pronóstico de los oligodendrogliomas frontales.

\section{Oligodendrogliomas anaplásicos frente a astrocitomas de grado IV}

Es importante diferenciar ambos tipos tumorales porque tienen pronósticos distintos. La diferenciación histopatológica entre ambos tumores es posible y objetiva en gran parte de los casos. Sin embargo, en circunstancias especiales, el diagnóstico diferencial resulta difícil. En esta tesitura es de utilidad el estudio de la deleción 1p y 19q. Es posible que en el futuro puedan obtenerse micromatrices de ADN útiles en el diagnóstico diferencial entre ambos tipos tumorales.

\section{OLIGOASTROCITOMAS Características generales}

Se denominan oligoastrocitomas aquellos tumores que presentan un doble componente, oligodendroglial y astroglial, que puede estar o no entremezclado. Es por tanto un tipo tumoral muy heterogéneo, a caballo entre el oligodendroglioma y el astrocitoma. Se distinguen varios subtipos histológicos: oligoastrocitomas de bajo grado (grado II) y oligoastrocitomas anaplásicos (grado III).

\section{Dinámica de malignización y factores pronósticos}

Como se podría deducir de los dos componentes tumorales integrantes, presentan rasgos similares a los indicados para los astrocitomas y oligodendrogliomas $[1,38]$.

\section{Aspectos moleculares}

El diagnóstico de los oligoastrocitomas plantea grandes dificultades. Se ha mostrado útil el estudio de la deleción combinada $1 \mathrm{p} / 19 \mathrm{q}$, de tal manera que los oligoastrocitomas cerebrales no temporales muestran esta deleción, como los oligodendrogliomas convencionales. Sin embargo, los oligoastrocitomas temporales no presentan deleción combinada y, además, muestran mutación del gen TP53 [37].

\section{EPENDIMOMAS \\ Características generales}

Representan el 3-9\% de todos los tumores neuroepiteliales. Se distinguen cuatro subtipos histológicos: clásico, anaplásico, mixopapilar y subependimoma.

\section{Dinámica de la malignización del ependimoma}

Los ependimomas de bajo grado muestran tendencia a transformarse en ependimomas anaplásicos, aunque no existe una estimación de dicha tendencia.

La gradación histopatológica de los gliomas es útil. Se considera de grado I (OMS) el subependimoma y el ependimoma mixopapilar; de grado II, el ependimoma de bajo grado, y de grado III, el ependimoma anaplásico. La supervivencia libre de enfermedad a los 10 años de los ependimomas clásicos es del 48\%, y para los anaplásicos, del $25 \%$.

\section{Aspectos moleculares de interés en los ependimomas}

Los aspectos moleculares más sobresalientes son:

- Marcadores de proliferación celular. La estimación de la inmunorreactividad frente al antígeno Ki-67 puede ser un marcador pronóstico en los ependimomas en general [39] y de los de la fosa posterior en particular [40].

- Hipermetilación de múltiples genes que podrían relacionarse con la oncogénesis de los ependimomas. También se ha detectado una frecuente pérdida del cromosoma 22, especialmente en los ependimomas espinales. No existen datos pronósticos de aplicación clínica en relación con la ploidía y las alteraciones citogenéticas y moleculares en los ependimomas. En trabajos recientes se apunta la posibilidad de que, en los ependimomas intracraneales, la ganancia de la región cromosómica 1q y la sobreexpresión del factor EGFR pueden ser factores pronósticos independientes [41]. 


\section{NOVEDADES SOBRE LA PREDICCIÓN DE RESPUESTA AL TRATAMIENTO DE LOS GLIOMAS Utilidad de la biología molecular en la valoración de la respuesta antitumoral: hallazgos iniciales}

No existen todavía hallazgos firmemente establecidos, sino aproximaciones. Las células de los astrocitomas de alto grado son en general quimiorradiorresistentes. Existen algunos trabajos recientes que establecen una asociación entre ciertas alteraciones génicas y la resistencia tumoral a los citostáticos:

- La inactivación del gen reparador del ADN, denominado $O^{6}$-MGMT (metil-guanil-metil-transferasa), por hipermetilación del promotor se asocia a la respuesta al tratamiento mediante agentes alquilantes. Así, Esteller et al [42] concluyeron que la resistencia tumoral al tratamiento por los agentes alquilantes se asocia a hipermetilación del enzima MGMT, que se localiza en la región cromosómica 10q26. Estos resultados han sido avalados por otros grupos [43]. Se ha defendido que el estado de metilación del promotor del gen del enzima MGMT predice la respuesta al tratamiento del tumor con BCNU. Es más, es posible que el estado de metilación del promotor del gen tumoral puede evaluarse mediante el ADN sérico [44]. Son necesarios más estudios para conocer la relevancia clínica de la evaluación de la inactivación del enzima MGMT como predictor de respuesta al tratamiento en el glioblastoma y, de momento, no está justificada su aplicación rutinaria en la práctica clínica.

- La radiorresistencia y la resistencia de las células tumorales a ciertos citostáticos se asocia a la inactivación del gen TP53 $[45,46]$.

- El efecto citostático del BCNU puede estar en relación con el factor de necrosis tumoral. Este hecho se ha observado en líneas celulares de glioblastoma en el que las líneas respondedoras mostraban una marcada inducción de dicho factor, a diferencia de las que eran refractarias al tratamiento [47].
- Como se ha indicado anteriormente, existe una relación entre la deleción combinada 1p/19q y la respuesta al tratamiento en el oligodendroglioma anaplásico. Varios grupos de investigación han demostrado que los oligodendrogliomas anaplásicos con deleciones en el cromosoma 1p y 19q (ambas alteraciones tienden a producirse simultáneamente [48]) responden mejor al tratamiento con procarbacina, ciclofosfamida (Citoxan ${ }^{\circledR}$ ) y vincristina que los que no muestran estas alteraciones moleculares [49]. Este es el dato procedente de la citogenética molecular de los tumores cerebrales que ha demostrado un mayor impacto clínico hasta el momento.

\section{CONCLUSIÓN}

Podría resultar desalentador comprobar que de la investigación molecular desarrollada hasta la fecha sólo se han generado unos pocos factores moleculares de utilidad clínica. Una de las razones de este aparente fracaso es la metodológica. Se ha llevado a cabo hasta ahora una investigación de unos pocos genes definidos de antemano, pero no un estudio multigénico, especialmente de los genes implicados en propiedades biológicas clave, con la moderna técnica de la genómica (micromatrices). Es necesario diseñar estudios que permitan probar subgrupos de pacientes con gliomas tratados de manera similar, bien caracterizados, en los que se haya considerado la heterogeneidad tumoral y de quienes se disponga de un completo seguimiento clínico. Ésta es una de las tareas pendientes, de la que debería surgir una nueva clasificación de los tumores basada en los hallazgos moleculares, que no sustituirá sino que complementará la actual.

El futuro de la investigación molecular aplicada al diagnóstico y tratamiento de los gliomas es esperanzador, pero requiere una gran dosis de originalidad. Tampoco se conseguirá nada valioso si la investigación no se hace por equipos pluridisciplinares integrados por investigadores audaces y creativos.

\section{BIBLIOGRAFÍA}

1. Kleihues P, Cavenee WP. Pathology and genetics of tumors of the nervous system. Lyon: IARC Press; 2000.

2. Leenstra S, Oskam NT, Bijleveld EH, Bosch DA, Troost D, Hulsebos TJ. Genetic sub-types of human malignant astrocytoma correlate with survival. Int J Cancer 1998; 79: 159-65.

3. Steilen-Gimbel H, Steudel WI, Feiden W, Moringlane JR, Henn W, Zang KD. Genetic heterogeneity in human astrocytomas: spatial distribution of P16 and TP53 deletions in biopsies. Cancer Genet Cytogenet 1999; 113: 115-9.

4. Jung V, Romeike BF, Henn W, Feiden W, Moringlane JR, Zang KD, et al. Evidence of focal genetic microheterogeneity in glioblastoma multiforme by area-specific CGH on microdissected tumor cells. J Neuropathol Exp Neurol 1999; 58: 993-9.

5. Malmer B, Iselius L, Holmberg E, Collins A, Henriksson R, Gronberg H. Genetic epidemiology of glioma. Br J Cancer 2001; 84: 429-34.

6. Cheng Y, Ng HK, Ding M, Zhang SF, Pang JC, Lo KW. Molecular analysis of microdissected de novo glioblastomas and paired astrocytic tumors. J Neuropathol Exp Neurol 1999; 58: 120-8.

7. Heinmoller E, Liu Q, Sun Y, Schlake G, Hill KA, Weiss LM, et al. Toward efficient analysis of mutations in single cells from ethanolfixed, paraffin-embedded, and immunohistochemically stained tissues. Lab Invest 2002; 82: 443-53.

8. Alcock HE, Stephenson TJ, Royds JA, Hammond DW. A simple method for PCR based analyses of immunohistochemically stained, microdissected, formalin fixed, paraffin wax embedded material. Mol Pathol 1999; 52: 160-3.

9. Watanabe K, Tachibana O, Yonekawa Y, Kleihues P, Ohgaki H. Role of gemistocytes in astrocytoma progression. Lab Invest 1997; 76: 277-84.

10. Jaros E, Perry RH, Adam L, Kelly PJ, Crawford PJ, Kalbag RM, et al. Prognostic implications of p53 protein, epidermal growth factor receptor, and Ki-67 labelling in brain tumours. Br J Cancer 1992; 66: 373-85.
11. Montine TJ, Vandersteenhoven JJ, Aguzzi A, Boyko OB, Dodge RK, Kerns BJ, et al. Prognostic significance of Ki-67 proliferation index in supratentorial fibrillary astrocytic neoplasms. Neurosurgery 1994; 34 : 674-9.

12. Hsu DW, Louis DN, Efird JT, Hedley-Whyte ET. Use of MIB-1 (Ki67 immunoreactivity in differentiating grade II and grade III gliomas. J Neuropathol Exp Neurol 1997; 56: 857-65.

13. Coons SW, Davis JR, Way DL. Correlation of DNA content and histology in prognosis of astrocytomas. Am J Clin Pathol 1988; 90: 289-93.

14. Van Meyel DJ, Ramsay DA, Casson AG, Keeney M, Chambers AF, Cairncross JG. p53 mutation, expression, and DNA ploidy in evolving gliomas: evidence for two pathways of progression. J Natl Cancer Inst 1994; 86: 1011-7.

15. Louis DN. A molecular genetic model of astrocytoma histopathology. Brain Pathol 1997; 7: 755-64.

16. Newcomb EW, Cohen H, Lee SR, Bhalla SK, Bloom J, Hayes RL, et al. Survival of patients with glioblastoma multiforme is not influenced by altered expression of $p 16, p 53, E G F R, M D M 2$ or $B c l-2$ genes. Brain Pathol 1998; 8: 655-67.

17. Puduvalli VK, Kyritsis AP, Hess KR, Bondy ML, Fuller GN, Kouraklis $\mathrm{GP}$, et al. Patterns of expression of $\mathrm{Rb}$ and p16 in astrocytic gliomas, and correlation with survival. Int J Oncol 2000; 17: 963-9.

18. Watanabe K, Sato K, Biernat W, Tachibana O, Von Ammon K, Ogata $\mathrm{N}$, et al. Incidence and timing of p53 mutations during astrocytoma progression in patients with multiple biopsies. Clin Cancer Res 1997; 3: 523-30.

19. Prives C, Hall PA. The p53 pathway. J Pathol 1999; 187: 112-26.

20. Lang FF, Miller DC, Pisharody S, Koslow M, Newcomb EW. High frequency of $\mathrm{p} 53$ protein accumulation without $p 53$ gene mutation in human juvenile pilocytic, low grade and anaplastic astrocytomas. Oncogene 1994; 9: 949-54. 
21. Nakamura M, Konishi N, Tsunoda S, Nakase H, Tsuzuki T, Aoki H, et al. Analysis of prognostic and survival factors related to treatment of low-grade astrocytomas in adults. Oncology 2000; 58: 108-16.

22. Kraus JA, Bolln C, Wolf HK, Neumann J, Kindermann D, Fimmers R, et al. TP53 alterations and clinical outcome in low grade astrocytomas. Genes Chromosomes Cancer 1994; 10: 143-9.

23. Sallinen SL, Sallinen P, Haapasalo H, Kononen J, Karhu R, Helen P, et al. Accumulation of genetic changes is associated with poor prognosis in grade II astrocytomas. Am J Pathol 1997; 151: 1799-807.

24. Ohgaki H, Dessen P, Jourde B, Horstmann S, Nishikawa T, Di Patre PL, et al. Genetic pathways to glioblastoma: a population-based study. Cancer Res 2004; 64: 6892-9.

25. Idoate M, Sola I, Manrique M, De Álava E, Pardo F. Loss of heterozigosity of DCC region (18q21-23) and reduction of immunoreactivity of the corresponding protein are involved in astrocytoma malignization. Modern Pathol 2000; 13: 26A.

26. Terada K, Tamiya T, Daido S, Kambara H, Tanaka H, Ono Y, et al. Prognostic value of loss of heterozygosity around three candidate tumor suppressor genes on chromosome $10 \mathrm{q}$ in astrocytomas. J Neurooncol 2002; 58: 107-14.

27. Korshunov A, Sycheva R, Golanov A. The prognostic relevance of molecular alterations in glioblastomas for patients age $<50$ years. Cancer 2005; 104: 825-32.

28. Idoate MA, Soria E, Lozano MD, Sola JJ, Panizo A, De Álava E, et al. PTEN protein expression correlates with PTEN gene molecular changes but not with VEGF expression in astrocytomas. Diagn Mol Pathol 2003; 12: $160-5$

29. Idoate MA, Andión E, García-Foncillas J, Panizo A, Lozano MD, Toledo G, et al. Correlation between real time RT-PCR expression of PTEN and tumoral grade in astrocytomas. Modern Pathol 2005; 18: 292A.

30. Shaw EG, Scheithauer BW, O'Fallon JR, Davis DH. Mixed oligoastrocytomas: a survival and prognostic factor analysis. Neurosurgery 1994; 34: $577-82$.

31. Heesters M, Molenaar W, Go GK. Radiotherapy in supratentorial gliomas. A study of 821 cases. Strahlenther Onkol 2003; 179: 606-14.

32. Kros JM, Van Eden CG, Stefanko SZ, Waayer-Van Batenburg M, Van der Kwast TH. Prognostic implications of glial fibrillary acidic protein containing cell types in oligodendrogliomas. Cancer 1990; 66: 1204-12.

33. Kros JM, Hop WC, Godschalk JJ, Krishnadath KK. Prognostic value of the proliferation-related antigen Ki-67 in oligodendrogliomas. Cancer 1996; 78: 1107-13.

34. Kros JM, Van Eden CG, Vissers CJ, Mulder AH, Van der Kwast TH. Prognostic relevance of DNA flow cytometry in oligodendroglioma. Cancer 1992; 69: 1791-8.

35. Kros JM, Godschalk JJ, Krishnadath KK, Van Eden CG. Expression of p53 in oligodendrogliomas. J Pathol 1993; 171: 285-90.

36. Hagel C, Krog B, Laas R, Stavrou DK. Prognostic relevance of TP53 mutations, p53 protein, Ki-67 index and conventional histological grading in oligodendrogliomas. J Exp Clin Cancer Res 1999; 18: 305-9.
37. Mueller W, Hartmann C, Hoffmann A, Lanksch W, Kiwit J, Tonn J, et al. Genetic signature of oligoastrocytomas correlates with tumor location and denotes distinct molecular subsets. Am J Pathol 2002; 161: 313-9.

38. Tamura M, Zama A, Kurihara H, Kano T, Imai H, Ishiuchi S, et al. Clinicohistological study of oligodendroglioma and oligoastrocytoma. Brain Tumor Pathol 1997; 14: 35-9.

39. Ritter AM, Hess KR, McLendon RE, Langford LA. Ependymomas: MIB-1 proliferation index and survival. J Neurooncol 1998; 40: 51-7.

40. Bennetto L, Foreman N, Harding B, Hayward R, Ironside J, Love S, et al. $\mathrm{Ki}-67$ immunolabelling index is a prognostic indicator in childhood posterior fossa ependymomas. Neuropathol Appl Neurobiol 1998; 24: 434-40.

41. Mendrzyk F, Korshunov A, Benner A, Toedt G, Pfister S, Radlwimmer $\mathrm{B}$, et al. Identification of gains on $1 \mathrm{q}$ and epidermal growth factor receptor overexpression as independent prognostic markers in intracranial ependymoma. Clin Cancer Res 2006; 12: 2070-9.

42. Esteller M, García-Foncillas J, Andión E, Goodman SN, Hidalgo OF, Vanaclocha V, et al. Inactivation of the DNA-repair gene MGMT and the clinical response of gliomas to alkylating agents. NEngl J Med 2000; 343: 1350-4.

43. Nutt CL, Noble M, Chambers AF, Cairncross JG. Differential expression of drug resistance genes and chemosensitivity in glial cell lineages correlate with differential response of oligodendrogliomas and astrocytomas to chemotherapy. Cancer Res 2000; 60: 4812-8.

44. Balañá C, Ramírez JL, Taron M, Roussos Y, Ariza A, Ballester R, et al. $\mathrm{O}^{6}$-methyl-guanine-DNA methyltransferase methylation in serum and tumor DNA predicts response to 1,3-bis(2-chloroethyl)-1-nitrosourea but not to temozolamide plus cisplatin in glioblastoma multiforme. Clin Cancer Res 2003; 9: 1461-8.

45. Shu HK, Kim MM, Chen P, Furman F, Julin CM, Israel MA. The intrinsic radioresistance of glioblastoma-derived cell lines is associated with a failure of p53 to induce p21(BAX) expression. Proc Natl Acad Sci U S A 1998; 95: 14453-8.

46. O’Connor PM, Jackman J, Bae I, Myers TG, Fan S, Mutoh M, et al. Characterization of the p53 tumor suppressor pathway in cell lines of the National Cancer Institute anticancer drug screen and correlations with the growth-inhibitory potency of 123 anticancer agents. Cancer Res 1997; 57: 4285-300.

47. Rhee CH, Ruan S, Chen S, Chenchik A, Levin VA, Yung AW, et al. Characterization of cellular pathways involved in glioblastoma response to the chemotherapeutic agent 1,3-bis(2-chloroethyl)-1-nitrosourea (BCNU) by gene expression profiling. Oncol Rep 1999; 6: 393-401.

48. Smith JS, Perry A, Borell TJ, Lee HK, O'Fallon J, Hosek SM, et al. Alterations of chromosome arms $1 \mathrm{p}$ and $19 \mathrm{q}$ as predictors of survival in oligodendrogliomas, astrocytomas, and mixed oligoastrocytomas. J Clin Oncol 2000; 18: 636-45.

49. Cairncross JG, Ueki K, Zlatescu MC, Lisle DK, Finkelstein DM, Hammond RR, et al. Specific genetic predictors of chemotherapeutic response and survival in patients with anaplastic oligodendrogliomas. J Natl Cancer Inst 1998; 90: 1473-9.

\section{UPDATE ON THE MOLECULAR BIOLOGY OF GLIOMAS: \\ TOWARDS A PATHOMOLECULAR CLASSIFICATION OF GLIOMAS}

Summary. Aim. To review the current state of the classification and oncogenesis of gliomas, emphasizing those biologic parameters with special clinical significance. Development. In the current classification, both histologic grade and phenotype are considered the pathological features with more relevant clinical impact. These factors are an obligatory reference for both all molecular studies and a new classification. The relationship between the oligodendroglial phenotype and the loss of chromosomes $1 p$ and $19 q$ is a useful data in the histopathologic differential diagnosis. The new pathologic-molecular classification should take into account the current state of knowledge about the malignization pathways of gliomas, which have prognostic significance. The neoplastic biological potential should be determined in each case according with the tumoral heterogeneity. Then, this evaluation can be based on tumoral microdissection. Although no well established prognostic molecular profiles are available, several molecular alterations are relevant such as chromosome 10 deletion, especially of the 10q23 region, mutation of PTEN and TP53 genes and amplification or mutation of EGFR. For treatment purposes, the combined deletion $1 p / 19 q$ identifies the anaplastic type of oligodendrogliomas that are more responsive to chemothetrapy. Conclusions. The new pathomolecular classification of gliomas should improve the old one, especially being concerned about the oncogenesis and heterogeneity of these tumors. It is desirable that this classification has clinical applicability and can integrate new molecular findings with some known histological features with prognostic value. [REV NEUROL 2007; 44: 217-24]

Key words. Astrocytomas. Gliomas. Immunohistochemistry. Molecular alteration. Oncogenesis. Prognostic factors. 